\title{
1,1'-螺二氢狮-7,7'-二酚(SPINOL)及类似物中螺环骨架的构建方法
}

\author{
赵启航 ${ }^{\dagger}$ 唐珑畅 ${ }^{\dagger}$ 焦 鹏* \\ (北京师范大学化学学院 北京 100875)
}

\begin{abstract}
摘要 $1,1^{\prime}$-螺二氢狮骨架是在 21 世纪初发展起来的一类新型手性配体或催化剂的骨架，具有 $C_{2}$ 对称性，以及刚性强、 稳定性高、易于修饰等特点. 周其林等将手性 1,1'-螺二氢狮-7,7'-二酚(SPINOL)发展为一系列螺环配体及催化剂, 在催 化不对称合成领域获得了巨大的成功. 1,1'-螺二氢狮骨架的配体及催化剂被认为是为数不多的 “优势手性配体和催化 剂” . 尽管催化的不对称合成已经出现，目前方法得到的 SPINOL 及类似物多为外消旋体，需要进行费力的手性拆分. 合成和拆分步骤繁琐的局限性对其大规模生产形成了阻碍. 因此, 如何从廉价易得的原料出发, 发展相应手性螺环骨 架配体的高效、不对称催化合成新方法，是非常迫切而且具有挑战性的课题. 总结了最近 20 年 SPINOL 及螺环骨架类 似化合物的合成方法, 包括含杂原子的螺环骨架, 希望能对配体或催化剂用途的螺环骨架的构建有所启发.
\end{abstract}

关键词 1,1'-螺二氢狮-7,7'-二酚(SPINOL); 螺环骨架构建; $C_{2}$ 对称性配体; 手性拆分

\section{Construction of Spiro Skeletons in 2,2',3,3'-Tetrahydro-1,1'- spirobi[1H-indene]-7,7'-diol (SPINOL) and Analogues}

\author{
Zhao, Qihang $^{\dagger} \quad$ Tang, Longchang ${ }^{\dagger} \quad$ Jiao, Peng* \\ (College of Chemistry, Beijing Normal University, Beijing 100875)
}

\begin{abstract}
Spirobiindane possesses a new type of skeleton for chiral ligands developed in the early 21 st century, which has the characteristics of $C_{2}$-symmetry, strong rigidity, high stability, and ease of modification. Zhou et al. developed 2,2',3,3'-tetrahydro-1,1'-spirobi[1H-indene]-7,7'-diol (SPINOL) into series of chiral ligands and catalysts, and achieved great success in the field of catalytic asymmetric synthesis. These ligands and catalysts bearing 1,1'-spirobiindane skeleton are considered as few "privileged chiral ligands and catalysts". Though catalytic asymmetric syntheses have appeared, in the present methods SPINOL and analogues are usually obtained in racemic forms, which require further chiral resolution. Laborious synthetic and resolution steps limit their productions in industrial scale. Therefore, it is urgent as well as challenging to develop efficient method of asymmetric catalytic synthesis of chiral spiro ligands from cheap and readily available raw materials. The synthetic methods of SPINOL and analgoues in the past 20 years are summarized, including those bearing heteroatoms in the spiro skeleton, in the hope of sparking new ideas for the construction of spiro skeletons in ligands or catalysts.
\end{abstract}

Keywords 2,2',3,3'-tetrahydro-1,1'-spirobi[1H-indene]-7,7'-diol (SPINOL); construction of spiro skeleton; $C_{2}$-symmetric ligand; chiral resolution

1997 年，陈新滋、蒋耀忠等 ${ }^{[1]}$ 将手性螺 [4.4]壬烷1,6-二醇骨架的双膦配体(SpirOP)用于 $\mathrm{Rh}$ 催化的不对称 氢化反应, 是螺环骨架配体用于金属催化不对称反应的 先例. 之后, 丁奎岭等 ${ }^{[2]}$ 开发了基于螺[4.4]-1,6-壬二烯 骨架的 “膦-噁唑啉” (SpinPHOX)、双膦、双啞唑啉配 体, 并成功用于 $\mathrm{Ir}$ 催化的不对称氢化反应. 螺[4.4]壬烷 骨架不仅手性拆分困难，而且 1,6-位两个羟基距离过近，
导致基于这一骨架的优秀配体数量有限(图 1). 1,1'-螺二 氢狮骨架具有稳定的化学结构和刚性，在天然产物中存 在，部分衍生物具有药物活性并已实现人工合成和拆 分 ${ }^{[3]}$. 为形成有效的鳌合基团, Birman 课题组于 1999 年 首次成功合成了外消旋 1,1'-螺二氢狮-7,7'-二酚, 命名为 SPINOL $^{[4]}$, 并将对映体进行了拆分, 按中心手性命名了 SPINOL 对映体的绝对构型 ${ }^{[5]}$ (图 1).

\footnotetext{
* Corresponding author. E-mail: pjiao@bnu.edu.cn

Received March 28, 2021; revised June 1, 2021; published online June 15, 2021.

Project supported by the National Natural Science Foundation of China (No. 21875023).

国家自然科学基金(No. 21875023)资助项目.

†共同第一作者(These authors contributed equally to this work).
} 


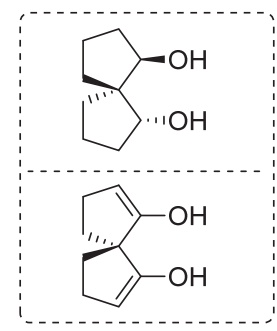

Less popular skeleton Hydroxy groups too close Enol form not readily accessible Ready for resolution and conversion

图 1 螺[4.4]壬烷-1,6-二醇/二酮与 SPINOL 的比较

Figure 1 Comparison of spiro[4.4]nonane-1,6-diol/dione with SPINOL

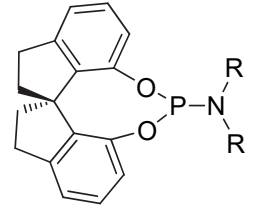

$(R)$-SIPHOS

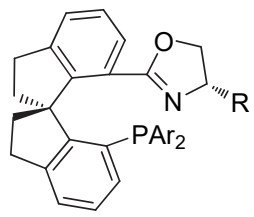

SIPHOX

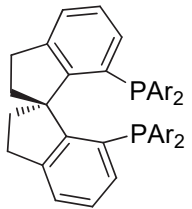

(R)-SDP

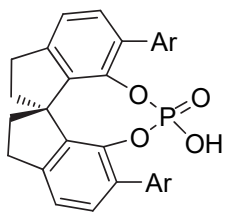

(S)-SPA
图 2 重要的 SPINOL 衍生物

Figure 2 Valuable SPINOL derivatives

2002 年, 周其林课题组 ${ }^{[6]}$ 以 SPINOL 为骨架, 设计 合成了手性螺环单膦配体 $N, N$-二甲基-(1,1'-螺二氢狮7,7'-二基)亚磷酰胺(SIPHOS), 并应用于铑催化的不对 称氢化反应中. 自此, 作为手性配体的 SPINOL 衍生物 (图 2)在过渡金属参与的不对称反应中表现卓越, 实现 了催化不对称氢化以及碳碳键偶联等高对映选择性转 化 ${ }^{[7]} .2010$ 年, SPINOL 衍生的手性磷酸(SPA)由林旭锋、 王彦广课题组 ${ }^{[8]}$ 设计合成, 应用于傅-克反应中. 如今, SPINOL 衍生的磷酸已被用于催化多种不对称合成反应 和动力学拆分 ${ }^{[8 b]}$. 周其林领导的 “高效手性螺环催化剂 的发现”项目获 2019 年国家自然科学奖一等奖, 这得益 于该课题组基于 SPINOL 骨架开发的一系列手性螺环配 体和催化剂在不对称合成中的卓越贡献.

经过国内外学者的广泛研究, SPINOL 骨架已成为 一类手性配体或催化剂的优势骨架(图 3). 但此优势骨 架的不对称合成依然具有挑战性. 迄今, 获得手性 SPINOL 主要依赖于借助手性试剂对外消旋体拆分的传 统方法, 特别是以羟基成酯为代表的成酯结晶法, 也有 少量催化的对映选择性合成方法见诸报道. 有关
SPINOL 螺环类似物的研究, 主要是为了发现更高效的 螺环骨架构建方法和性能更加优异的配体. 鉴于目前缺 乏对 SPINOL 及其类似物合成和拆分的综述，本文对到 目前为止成功合成、拆分 SPINOL 及其类似物的方法进 行了简单综述，并对未来寻找更加高效的合成方法作出 展望.

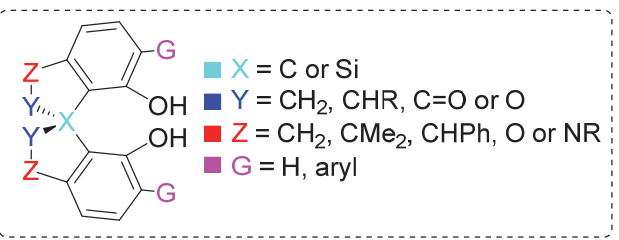

图 3 SPINOL 类似物

Figure 3 SPINOL analogues

\section{1 螺二氢狮骨架的二酚}

\section{1 螺二氢狮二酚(SPINOL)}

1999 年, Birman 等 ${ }^{[4]}$ 首次合成并以 $L$-氯甲酸薄荷酯 为拆分试剂得到光学纯的 SPINOL. Birman 的合成路线 (Scheme 1)是从 3-甲氧基苯甲醛(1)与丙酮出发, 羟醛缩 合产物在雷尼镍作用下催化氢化得到 1,5-二(3-甲氧基 苯基)-3-戊酮，在甲氧基对位溴化后得到 1,5-二(2-澳-5甲氧基苯基)-3-戊酮(2), 2 在多聚磷酸(PPA)催化下分子 内脱水环化得到外消旋的 SPINOL 3，总产率为 $28 \%$. 这 种环化方式是之后形成 SPINOL 骨架的主要方式. 在对 映体拆分中使用的 $L$-氯甲酸薄荷酯 D1 是一种对 SPINOL 极佳的手性拆分试剂: 在 $\mathrm{Et}_{3} \mathrm{~N}$ 和 4-二甲氨基吡 啶(DMAP)作用下得到一对非对映异构体，并通过重结 晶将二者分离，除去薄荷醇基团，最后在沸腾的己烷中 重结晶, 对 $(S)$-(一)-SPINOL 实现了 $91 \%$ 的产率, 对 $(R)$ $(+)$-SPINOL 实现了 $86 \%$ 的产率. 之后, 2004 年万伯顺 等 ${ }^{[9]}$ 同样使用了 $L$-氯甲酸薄荷酯, 并调整了溶剂及其他 条件，对 DBSPINOL (4)进行拆分. 在四丁基溴化铵 (TBAB) 存在下, 在 $0{ }^{\circ} \mathrm{C}$ 的 $\mathrm{NaOH} / \mathrm{CH}_{2} \mathrm{Cl}_{2}$ 混合液中与 $L-$ 氯甲酸薄荷酯成酯，实现了对 DBSPINOL 的拆分. (S)$(+)$-DBSPINOL (4) 的产率为 $98 \%$, 并且 $e e$ 值达到了 $99 \%$. 前述经典拆分方法中, 手性拆分试剂的化学计量 使用是 SPINOL 大规模生产的不利因素, 有必要开发更 为有效的合成与拆分方法. 对手性 SPINOL 获得方法的 改进，主要集中在外消旋体的拆分与催化不对称合成两 个方面.

同样具有 $C_{2}$ 对称性和二酚结构的手性 BINOL 及其 衍生物已经广泛用于催化和化学计量的不对称合成. 有 关 BINOL 已有许多成熟的拆分方法见诸报道 ${ }^{[10]}$, 这些 方法对获得手性 SPINOL 有一定的借鉴价值. 一些适用 


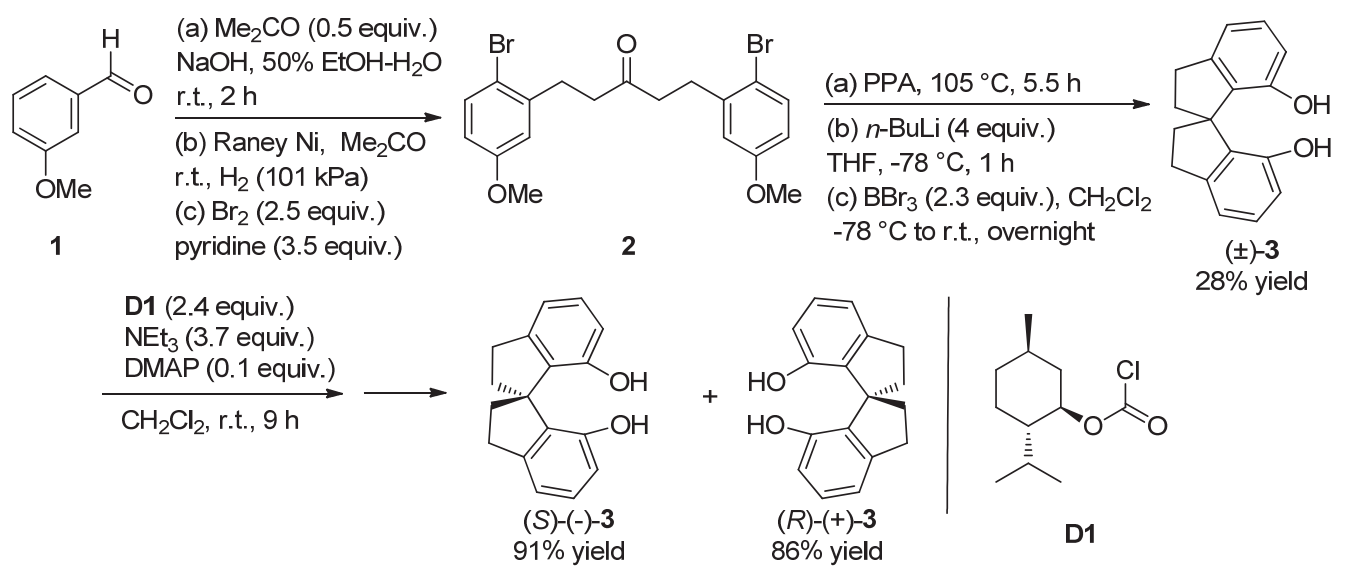

图式 1 Birman 的 SPINOL 合成及拆分路线

Scheme1 SPINOL synthesis and separation by Birman

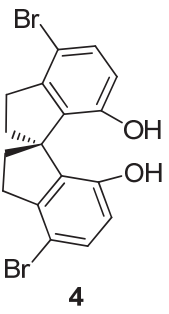

于 BINOL 的拆分方式同样适用于 SPINOL. 2002 年, 邓 金根、周其林、页涛等 ${ }^{[11]}$ 采用了 BINOL 拆分中常用的 包合结晶法, 将 $N$-芐基氯化辛可尼丁(D2)与外消旋的 SPINOL 以 $0.55: 1$ 的物质的量比共结晶得到包合物, 在甲苯中将 $(S)$-SPINOL 的包合物与未包合的 $(R)$-SPINOL 拆分, 包合物分解后在乙酸乙酯-己烷中重结晶, 最终以 $82 \%$ 的收率及 $75 \%$ 的收率分别获得大于 $99 \% \mathrm{ee}$ 值的(S)-(一)-SPINOL 和 $(R)-(+)$-SPINOL. 借助 $N$-芐基 氯化辛可尼丁的包合结晶法, 拆分效率高、SPINOL 纯 度高. 包合剂 D2 可方便地回收利用, 弥补了使用量大 的不足.<smiles>C=CC1CN(Cc2ccccc2)CC1C(O)c1ccnc2ccccc12</smiles>

另一方面, 如 Birman 等 ${ }^{[4]}$ 在报道中所指出的, 某些 适用于 BINOL 的拆分方式对 SPINOL 并不适用. 樟脑磺 酰氯或手性苯乙胺/硼酸无法用于 SPINOL 的拆分(图 4a). 2014 年, 赵宇等 ${ }^{[12]}$ 通过比较实验后指出, 适用于 BINOL 的氮杂环卡宾 (NHC) 催化的对映选择性酰化的 动力学拆分方法不适合于 SPINOL(图 4b). NHC 用于 BINOL 的动力学拆分, 能以 $>99 \%$ ee 值立体选择性地
获得轴手性产物. 这可能是因为在 BINOL 中酚羟基间 氢键的形成增加了反应物的亲核性，且有利于控制 BINOL 的构象. 而 SPINOL 骨架具有很大的刚性，在提 升其作为配体的手性诱导效应的同时，也加大了酚羟基 间生成氢键的难度，因而不能使用 NHC 进行拆分. 这 表明 SPINOL 结构的特殊性给其手性拆分的研究带来了 挑战.

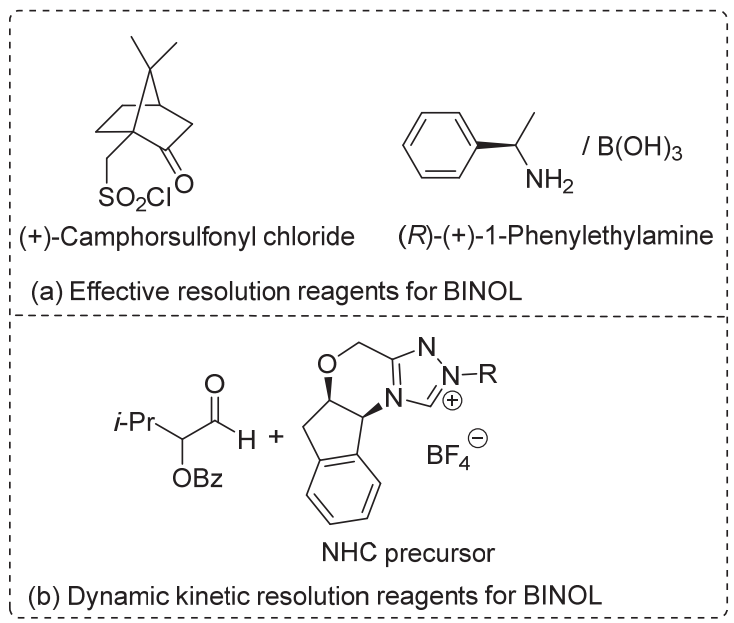

图 4 不适用于 SPINOL 的拆分试剂

Figure 4 Split reagents that proved to be unavailable for SPINOL

以上所述获得对映体纯 SPINOL 的方法, 主要依赖 于对 SPINOL 转化得来的非对映异构体的拆分，以及通 过包合结晶对 SPINOL 外消旋体的拆分. SPINOL 手性 季碳中心的直接构建鲜有报道. 在傅一克反应成环方式 的基础上，找到合适的手性催化剂是解决 SPINOL 不对 称合成的思路之一. Brønsted 酸催化剂对不对称合成有 着重要意义. 其中, 手性磷酸(CPA), 包括基于 BINOL、 VAPOL 和 SPINOL 等骨架的手性磷酸, 具有协同作用 的酸和碱双功能团可活化反应物，已被证实可用于催化 
一系列不对称反应 ${ }^{[13]}$. 基于此背景, 2016 年谭斌等 ${ }^{[14]}$ 在 篮选一系列 CPA 之后, 将 6,6'-位芳基取代的 SPINOL 衍 生的手性磷酸(SPA) C1 应用到 SPINOL 关键的成环步骤 中, 从缩酮出发首次实现了 SPINOL 衍生物的直接催化 对映选择性合成(Eq. 1). 使用 SPINOL 骨架的手性磷酸 催化, 可以得到与催化剂构型相反的单一对映异构体产 物. 该方法适用于多种类型的取代缩酮. 其中, 酚羟基 对位为吸电子取代基时, 产物的对映选择性更佳. 由 $\mathbf{5}$ 出发, 在 $3 \mathrm{~mol} \%$ 的催化剂用量下以三氯甲烷为溶剂, 在 $80{ }^{\circ} \mathrm{C}$ 反应 $8 \mathrm{~d}$ 得到 4 , 经重结晶 $e e$ 值可达 $99 \% .4$ 在 $\mathrm{Pd} / \mathrm{C}$ 催化下脱溴得到 $(S)$-SPINOL, 对映选择性保持不变, 这 是 SPINOL 不对称合成的重大进展. 此方法中使用的手 性 SPA 虽然价格昂贵, 但用量可低至 $0.1 \mathrm{~mol} \% .2018$ 年 林旭锋课题组 ${ }^{[15]}$ 在报道中指出, SPA 骨架刚性很强, 不 易消旋, 在反应结束后能够回收利用, 使得该方法具有 大规模应用的前景.<smiles>COCC(CCc1cc(O)ccc1Br)(CCc1cc(O)ccc1Br)OC</smiles>

5

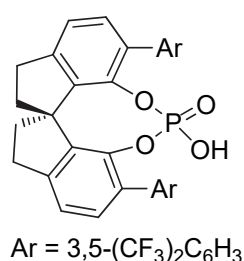

C1

\section{$1.22,2^{\prime}-/ 3,3^{\prime}-$-位修饰的 SPINOL 衍生物}

对手性配体的结构进行细微修饰, 不仅可以增强这 些配体在不对称催化中的多种功能, 而且新基团引入所 带来的手性中心, 会通过手性诱导效应或位阻效应对螺
环骨架合成时的立体选择性产生影响. 此外，还会带来 新的合成方法. 因此, 在 SPINOL 骨架的基础上, 发展 了一系列 2,2'-/3,3'-位取代基修饰的 SPINOL 衍生物, 带 来了螺环骨架对映选择性合成的新思路.

2004 年, Venugopal 等 ${ }^{[16]}$ 首次以 $40 \%$ 的产率合成了 4,4'-位溴代的环已基稠合螺二氢狮二酚 (CHEXDBSPINOL) (Scheme 2). 从 3-甲氧基苯甲醛(1)和环己酮出 发合成 7, 使用雷尼镍催化氢化后, 由 8 进一步环化合 成了外消旋 CHEXDBSPINOL (9). 由于 1,1',2,2'-位稠合 环已基的引入, 增加了螺环的刚性和位阻, 使得在螺环 合成过程中产生了立体控制. 在多聚磷酸(PPA)催化环 合时，直接得到了一对对映异构体的混合物，即 $(S, S, S)$ 9 和 $(R, R, R)-9$. 产物中螺环中心碳的绝对构型与 2,2'-位 两个手性中心的绝对构型产生了关联. 当 2,2'-位两个手 性中心都是 $(R)$-构型时, 螺环中心碳也是 $(R)$-构型. 进一 步用氯甲酸薄荷酯 D1 进行拆分, 分别得到了 $(S, S, S)$ $(+)$-CHEXDBSPINOL 和 $(R, R, R)-(-)$-CHEXDBSPINOL. 受到 2,2'-位相同构型的手性中心能诱导产生螺环 中心手性碳的启发, 丁奎岭等 ${ }^{[17]}$ 研究了 PPA 催化下 $\alpha, \alpha^{\prime}-$ 位双取代的外消旋戊酮衍生物的环化反应(Scheme 3), 由反式 $\alpha, \alpha^{\prime}$-二甲基酮 10 得到了一对对映异构体的外消 旋混合物 11. 遗憾的是, 没有找到有效的拆分外消旋混 合物 11 的试剂, 借助手性高效液相色谱(HPLC)进行拆 分, 才得到了一系列对映体纯 2,2'-位取代的 SPINOL 衍 生物. 在此尝试中, 不仅验证了 $2,2^{\prime}$-位的手性中心对成 环时螺环中心的手性有诱导作用, 更重要的是, 由于 2,2'-位取代基对两个苯环二面角及两个酚羟基氧原子间 距离产生影响, 配体的性质也相较于 SPINOL 时发生了 变化. 在 2,2'-位取代的 SPINOL 骨架上开发的手性单亚 磷酰胺配体在铑催化不对称氢化及铑催化对映选择性 $[4+2]$ 环加成反应中具有优异的性能. 这表明此类骨架

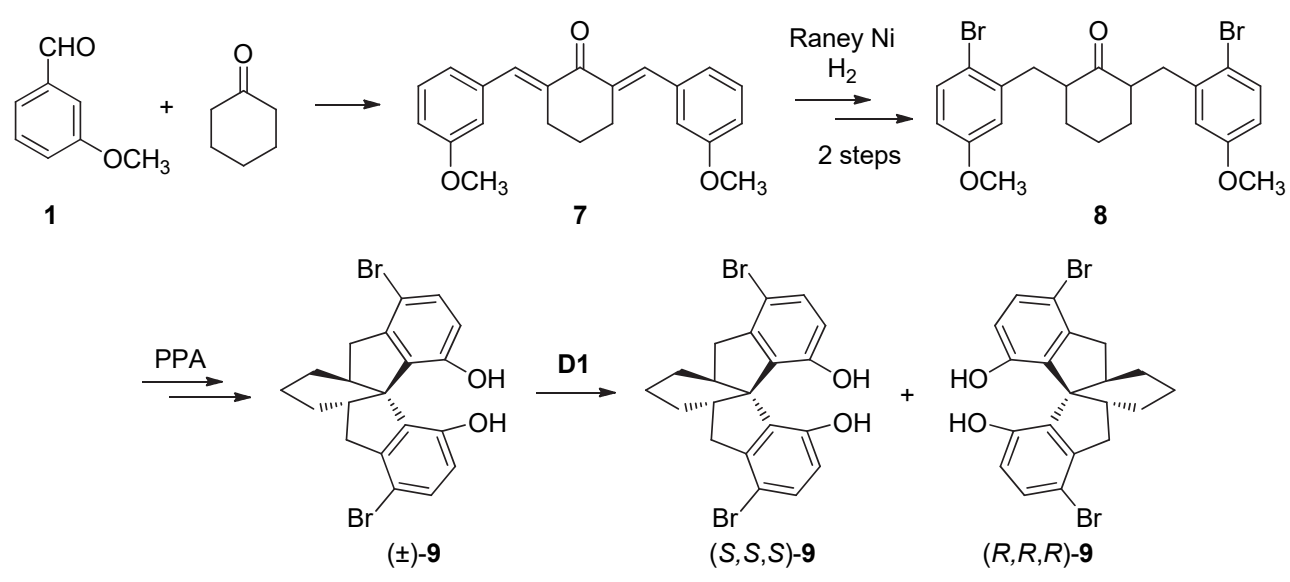

图式 2 CHEXDBSPINOL 的合成及拆分

Scheme 2 Synthesis and resolution of CHEXDBSPINOL 
<smiles>COc1ccc(Br)c(C[C@@H](C)C(=O)[C@H](C)Cc2cc(OC)ccc2Br)c1</smiles>

(土)-trans-10

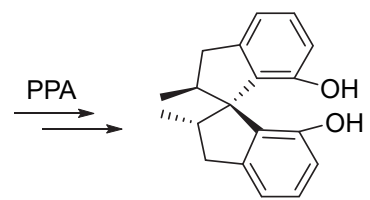

$( \pm)-11$
图式 32 2,2'-位取代的 SPINOL 衍生物 11 的合成 Scheme 3 Synthesis of 2,2'-substituted SPINOL derivative 11

具有开发的潜力. 虽然上述尝试是由类似 8、10 的外消 旋化合物在 PPA 作用下形成 2,2'-取代的外消旋 SPINOL 衍生物, 但是丁奎岭等在此基础上进一步的探索中, 找 到了合适的成环反应的试剂与条件，借助成环前分子内 的手性诱导或位阻效应, 直接得到单一手性的螺环产 物.

2018 年, 丁奎岭等 ${ }^{[18]}$ 在合成 CHEXDBSPINOL 时 (Scheme 4), 区别于 Venugopa 等 ${ }^{[16]}$ 的雷尼镍催化氢化, 开创性地利用 $1.0 \mathrm{~mol} \%$ 铱催化剂 $\operatorname{Ir}(\mathrm{I}) /(S)-{ }^{t} \mathrm{Bu}-\mathrm{PHOX}$, 在 室温下对 $\alpha, \beta$-不饱和酮 12 的环外双键进行不对称氢化, 最终以 90\%收率、良好的非对映异构体选择性(>20:1 $d r)$ 、优异的对映异构体选择性( $99 \%$ ee ) 得到手性中心位
于羰基 $\alpha$-位的环己酮 $\mathbf{8}$, 在螺环中心构建之前引入了手 性中心. 为避免 $\left(S, S^{\prime}\right)-\mathbf{8}$ 的分子中羰基邻位的手性中心 消旋化，在选择催化环化反应的酸催化剂时，未使用 SPINOL 合成时常用的 PPA, 而是篮选了一系列的 Brønsted 酸和 Lewis 酸. 最终使用强亲氧性 Lewis 酸 $\mathrm{TiCl}_{4}$ 进行催化，直接得到了单一对映体 9.9 脱溴后以 67\%的总产率得到环己基稠合螺环二酚 13 (chf-SPIN$\mathrm{OL}$, ee 值高达 $99 \%$. 丁奎岭等认为, $\mathrm{TiCl}_{4}$ 与酮羰基的 $\mathrm{O}$ 络合，增加了羰基的极性，从而促进了分子内傅-克反 应. 这一机理同时也导致 $\mathrm{TiCl}_{4}$ 使用量是化学计量的缺 点, 对大规模生产不利. 这个方法中, 在形成螺环之前, 利用不对称氢化反应预先引入了羰基 $\alpha$-位两个手性一 致的碳中心，并利用分子内的位阻效应以及合适的催化 剂, 实现了 SPINOL 单一手性的直接构建. 这对 SPINOL 骨架的不对称合成具有重大意义.

类似地，陆涛、Hayashi、窦晓巍等 ${ }^{[19]}$ 在 2019 年实 现了由手性酮出发通过催化不对称合成来构建手性螺 环中心(Scheme 5). 与丁奎岭等方法不同的是, 窦等利 用 $1 \mathrm{~mol} \%$ 的铑催化剂 $\mathbf{C 3}$ 在碱性条件下催化苯基硼酸

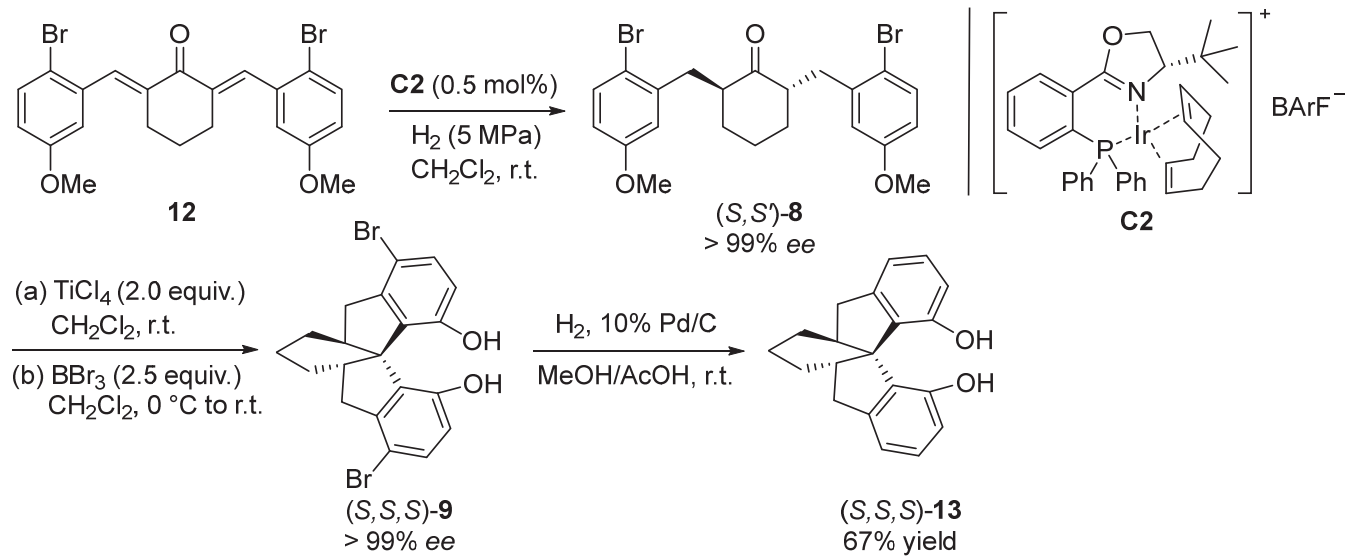

图式 4 CHEXDBSPINOL 的对映选择性合成

Scheme 4 Enantioselective synthesis of CHEXDBSPINOL<smiles>O=C(/C=C/c1cc(O)ccc1Br)/C=C/c1cc(O)ccc1Br</smiles>

C3 (1 mol\% Rh) $\underset{\mathrm{PhB}(\mathrm{OH})_{2}}{\stackrel{\mathrm{KOH}(5 \mathrm{~mol} \%)}{\longrightarrow}}$ toluene/ $\mathrm{H}_{2} \mathrm{O}$ $60^{\circ} \mathrm{C}, 12 \mathrm{~h}$<smiles>O=C(CC(c1ccccc1)c1cc(O)ccc1Br)CC(c1ccccc1)c1cc(O)ccc1Br</smiles>

15

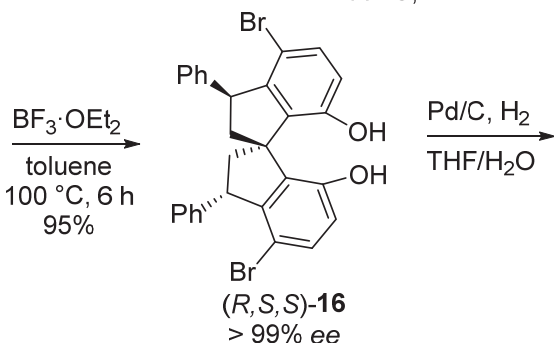

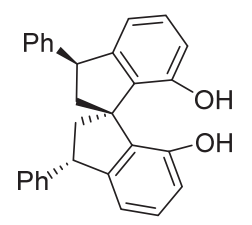

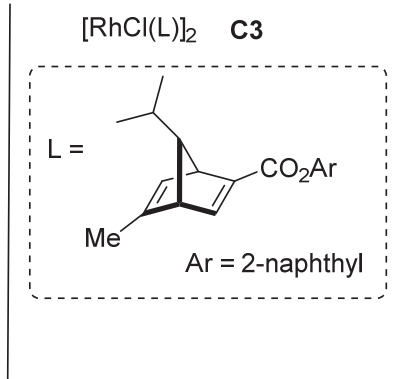

$(R, S, S)-17$ $90 \%$ yield

图式 $53,3^{\prime}$-位取代的 SPINOL 衍生物 17 的对映选择性合成

Scheme 5 Enantioselective synthesis of 3,3'-substituted SPINOL derivative 17 
对 $\alpha, \beta$-不饱和酮 14 进行不对称加成, 在羰基 $\beta$-位引入大 位阻芳香取代基, 得到手性中心位于羰基 $\beta$-位的酮 15 . 之后, 利用三氟化硼乙醚络合物促进分子内环化反应, 直接得到了含手性季碳中心的产物 $\mathbf{1 6}$, 产率达到了 95\% (>99\%ee). 在催化脱溴后, 实现了克级合成 3,3'位取代的 SPINOL 衍生物 17. 17 代表了一种优势的 SPINOL 衍生骨架, 由 $\mathbf{1 7}$ 合成的亚磷酰胺配体在不对称 催化中表现出高对映选择性. 窦晓巍认为, 17 相较于 SPINOL 的变化, 来自于 3,3'-位芳香取代基与羟基在空 间的分布. 3,3'-位芳香取代基的引入, 对于形成螺环步 骤的立体选择性及螺环骨架配体催化反应时的对映选 择性都至关重要. 并且, 3,3'-位手性中心的立体构型相 较于 2,2'-位手性中心更为稳定, 这对 SPINOL 衍生物的 设计合成具有指导意义.

经典的 SPINOL 螺环骨架的构建方法主要依赖于由 戊酮出发、以 PPA 作催化剂的分子内脱水环化反应. 前 述的几例报道便是如此. 利用预先引入取代基和手性中 心的策略, 对成环步的立体选择性加以控制, 实现了 SPINOL 类似物单一对映体的高产率克级合成. 以下尝 试则是对传统 SPINOL 合成方法的有益补充.

2018 年, 林旭锋 ${ }^{[20]}$ 等报道了以双酚 C 18 作原料、 在甲磺酸催化下通过分解、环化形成螺环骨架产物 6,6'-HMSIOL 19 的反应, 收率达到 92\% (Scheme 6). 该 反应能在 $20 \mathrm{~g}$ 规模一步完成, 重结晶即可获得纯的目标 产物. 在 19 的 7,7'-位进行溴代之后, 以 $(S)$-甲氧基芸丙 酰氯(D3)为拆分试剂进行传统手性拆分, 接着脱除 6,6'位酚羟基后得到二溴代化合物( $(R)-\mathbf{2 0}$, 并进一步转化为 螺环双膦配体 HMSI-PHOS (21). 21 被证明是 Pd 催化的 不对称合成的良好配体. 此外, 该课题组 ${ }^{[21]}$ 还报道了以 包合拆分试剂 $N$-芐基氯化辛可尼丁(D2)对 19 的大规模 拆分方法, D2 可以用简易的方法以 $>95 \%$ 的回收率回收 利用, 弥补了拆分剂使用量大的不足. 该课题组 ${ }^{[22]}$ 在同 年还报道了从双酚 A 22 出发合成 7,7'-TMSIOL (24)的方 法. 通过甲磺酸催化下双酚 A 的分解、环化反应 (Scheme 7), 以 89\%的产率获得 3,3,3',3'-四甲基-1,1'-螺 二氢苑-6,6'二酚的外消旋物 23. 对取代基进行转化, 在 7,7'-位引入羟基, 然后以传统的适用于 SPINOL 的氯甲 酸薄荷酯为拆分试剂, 以 $39 \%$ 的总收率得到对映体纯的 产物(S)-24. 与传统的 SPINOL 成环方式相比, 林旭锋报 道的方法在成环一步的产率大大提高. 虽然拆分仍使用 传统的手性试剂, 但所用原料便宜、易得, 该合成方法 已体现出明显的优势. 林旭锋的方法美中不足的是初始 螺环产物 19、23 中羟基位于 6,6'-位，而实际合成类似 SPINOL 骨架的配体时, 通常需要将 6,6'-位羟基脱除, 并且在 7,7'-位引入可转化利用的基团. 但同时, 也可以
巧妙地利用 6,6'-位的羟基进行官能团转化，制备很有价 值的 6,6'-位双取代的螺环二酚衍生物 ${ }^{[23]}$. 此外，双酚 $\mathrm{A}$ (双酚 $\mathrm{C}$ )的分解、环化反应(Scheme 7)，理论上从 3 分 子原料出发生成 4 分子苯酚(4 分子邻甲酚), 给 19、23 的纯化带来一定困难.
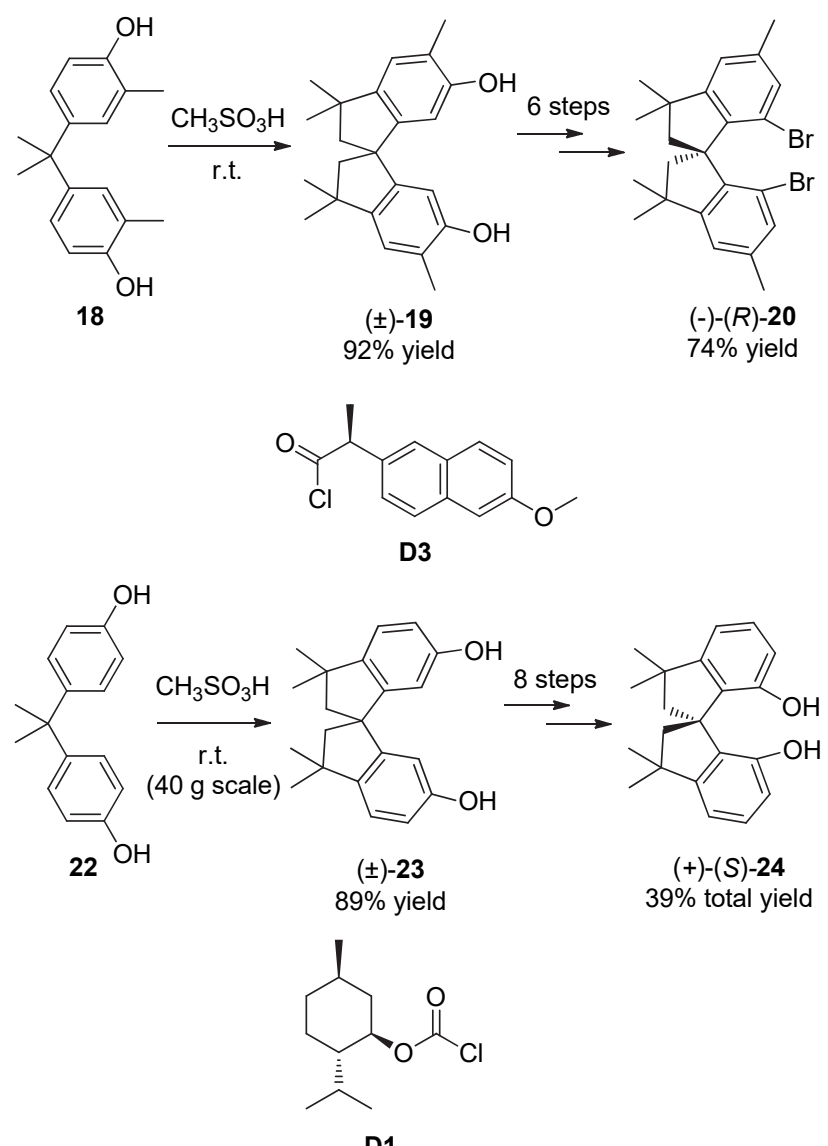

图式 6 由双酚 C 或双酚 A 出发的 SPINOL 类似物的合成 Scheme 6 Synthesis of SPINOL analogues from bisphenol C or bisphenol A

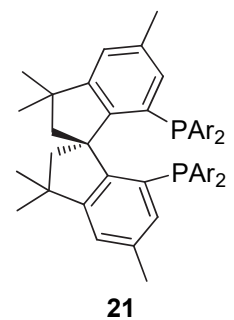

\section{2 螺环骨架含有杂原子的 SPINOL 类似物}

季碳中心是螺环骨架区别于其他刚性分子的重要 特征. 螺环季碳中心构建存在着许多经典方法，如分子 内双取代、自由基环化反应、分子内重排、环加成反应、 桥环体系的裂解等 ${ }^{[24]}$. 由于合成螺二氢狮骨架的配体 要将羟基基团的特定位置考虑在内, 这给合成螺环骨架 


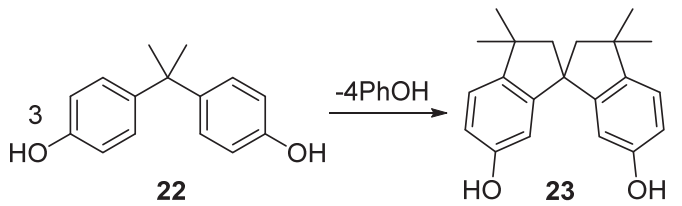

(a)

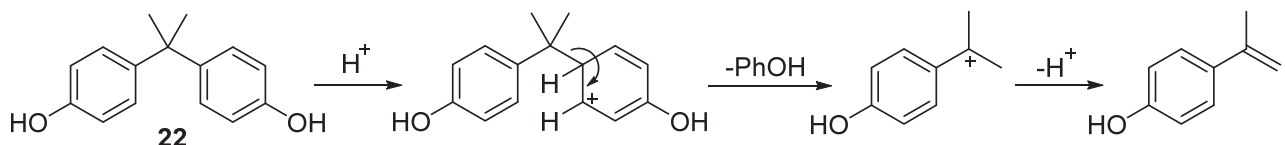

(b)

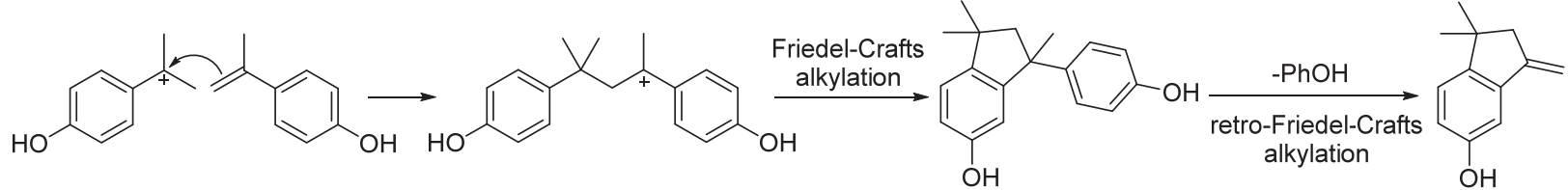

(c)<smiles>CC(C)(C)c1ccc(O)cc1</smiles><smiles>CC(C)(CCC1c2ccc(O)cc2C1(C)C)c1ccc(O)cc1</smiles>

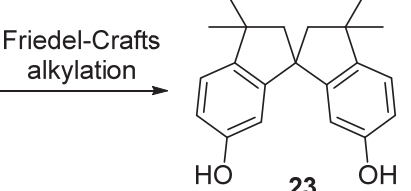

图式 7 由双酚 A 生成 23 的反应机理

Scheme 7 Reaction mechanism of the formation of $\mathbf{2 3}$ from bisphenol A

增加了难度，所用原料、合成方法都受到了限制. 螺环 骨架上杂原子的引入, 既可以在合成上采用更多的策 略, 杂原子与碳原子在电子性质以及空间性质方面的差 异, 使螺环骨架上含有杂原子的配体有可能在不对称催 化反应中产生不同的表现, 提高类螺二氢狮骨架的配体 的普适性. 杂原子存在于很多螺环结构中 ${ }^{[25]}$, 其合成方 法可为构建含杂原子的螺环骨架配体提供参考(图 5).

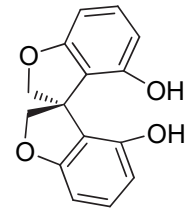

(S)-O-SPINOL

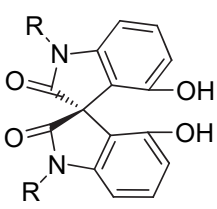

(S)-SBIDOL

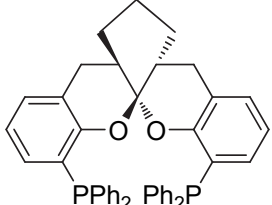

SKP

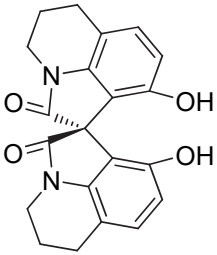

(S)-aza-SPINOL

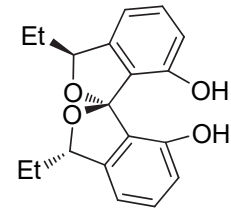

$(S, S, S)-S P I R O L$

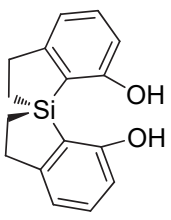

(S)-SPSiOL
图 5 含有杂原子的螺环骨架

Figure 5 Spiro skeletons bearing heteroatoms

\section{1 含氧 SPINOL 类似物}

2018 年, 张绪穆等 ${ }^{[26]}$ 合成了 $O$-SPINOL (Scheme 8a). 与传统的 SPINOL 合成时利用双分子内 FriedelCrafts 反应形成螺环的方式不同, $O$-SPINOL 的合成中先
引入了 25 分子中的季碳中心，接着通过芳香亲核取代 反应产生螺环结构, 得到 $r a c-26$, 经转化得到外消旋的 27. $L$-氯甲酸薄荷酯(D1)对 27 的拆分效果并不理想. 张 绪穆发现 $L$-脯氨酸(D4)在不同极性的溶剂中可对不同 构型的 27 进行手性识别: D4 在乙腈溶液中能够与 $(S)-27$ 有效地形成包结复合物, 而在乙酸乙酯溶液中则与 (R)-27 选择性结合. 包结复合物在超声辅助下于乙酸乙 酯和水的混合液中解离, 然后经过萃取得到光学活性产 物 $(R)-27$ 或 $(S)-27$ (ee 值达到 70\% 90\%). 以 $(S)-27$ 为骨 架发展的三齿手性螺环吡啶一胺基膦配体 L1, 已经应用 到酯的不对称催化氢化中，可实现 Bringmann 内酯的动 力学拆分. 配体 L1 在此反应中给出的产率和对映选择 性，不逊于 SPINOL 衍生的配体 L2 (Scheme 8b). 尽管 $O-S P I N O L$ 仍然需要通过拆分才能获得光学纯对映体, 但是通过对全碳螺环骨架的改造降低了原料成本. 而且 利用这种新颖的季碳中心构建方法，容易实现 $>100 \mathrm{~g}$ 规模的合成. 2018 年，蔡倩课题组 ${ }^{[27]}$ 报道了手性铜络合 物催化下碘苯与醇的偶联反应(Eq. 2), 经过两次分子内 碳氧偶联反应，可不对称地构建螺环。利用异丙醇重结 晶后, 能够得到光学纯度较高的螺环产物. 这种反应方 式有望继续发展为 $O$-SPINOL 不对称合成的新方法.

2003 年, van Leeuwen 等 ${ }^{[28]}$ 报道了螺二色烷骨架配 体 SPANphos (28) (Scheme 9)的合成. 他们利用对甲基 苯酚与丙酮的反应构建了缩酮结构的季碳中心. 对甲基 苯酚与丙酮反应生成的丙烯基取代产物，经过 DielsAlder 反应、retro-傅-克烷基化反应构建了螺缩酮骨架 
(a)<smiles>OCC(CO)(c1c(F)cccc1F)c1c(F)cccc1F</smiles>

25<smiles>Fc1cccc2c1C1(CO2)COc2cccc(F)c21</smiles>

rac-26<smiles>Oc1cccc2c1C1(CCO2)COc2cccc(O)c21</smiles>

rac-27

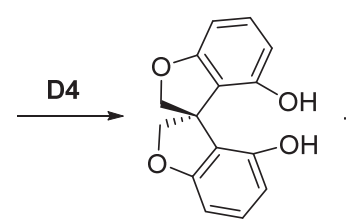

$(R)-27$

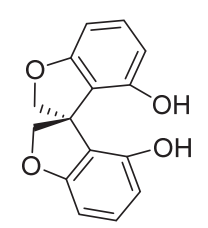

(S)-27

(b)<smiles>Cc1cccnc1CNc1cccc2c1[C@@]1(COc3cccc(P)c31)CO2</smiles>

L1
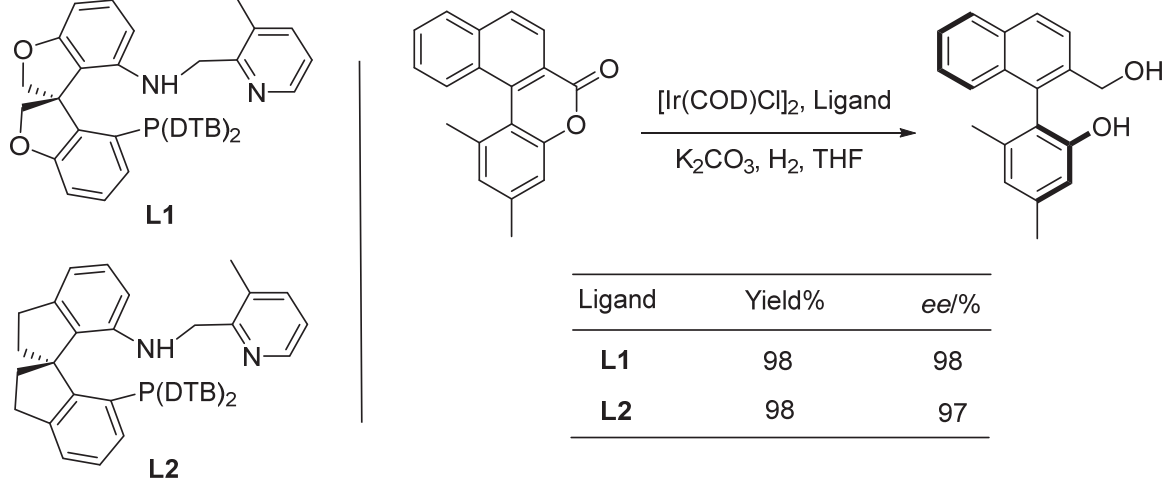

\begin{tabular}{ccc}
\hline Ligand & Yield\% & ee/\% \\
\hline L1 & 98 & 98 \\
L2 & 98 & 97 \\
\hline
\end{tabular}

图式 $8 O$-SPINOL 骨架手性配体的合成与应用

Scheme 8 Synthesis and application of a chiral ligand based on $O$-SPINOL<smiles>[X]C([Y]c1ccccc1I)(CO)CO</smiles>
$\underline{[\mathrm{Cu}] / \mathrm{L}}$

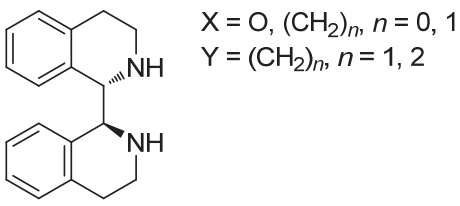

L

(Scheme 10). SPANphos 合成步骤少, 总收率达到了 $80 \%$, 并且原料相对廉价. 但当时课题组未找到合适的 方法将对映体拆分. SPANphos 被视为一种空间结构稳 定的反式双膦配体, 它与铑的配合物在甲醇羰基化制乙 酸的反应中表现出良好的催化活性, 转化数(TON)达到 902 [29]. 2012 年, van Leeuwen 等[30]用手性高效液相色谱 拆分得到了一系列光学活性的螺二色烷骨架的配体, 其 中 SPANphos 在钯催化的 $\alpha$-氰基苯乙酸酯的不对称氟化 反应中得到了良好的产率和对映选择性(Eq. 3). 由于分 子中没有化学拆分可用的基团, 导致对映体拆分困难, 这限制了螺二色烷骨架配体在不对称有机合成中的开 发。

2012 年, 丁奎岭等 ${ }^{2 \mathrm{~d}]}$ 利用 SpinPHOX/Ir(I)催化剂促 进 $\alpha, \alpha^{\prime}$-双(2-羟基苯亚甲基)环己酮(29)的不对称加氢和 加氢产物的螺缩酮化(Scheme 11), 以较高的对映选择性 和非对映选择性得到了螺二色烷骨架的分子 $\mathbf{3 0}$, 解决 了外消旋的螺二色烷类化合物手性拆分困难的问题. 不

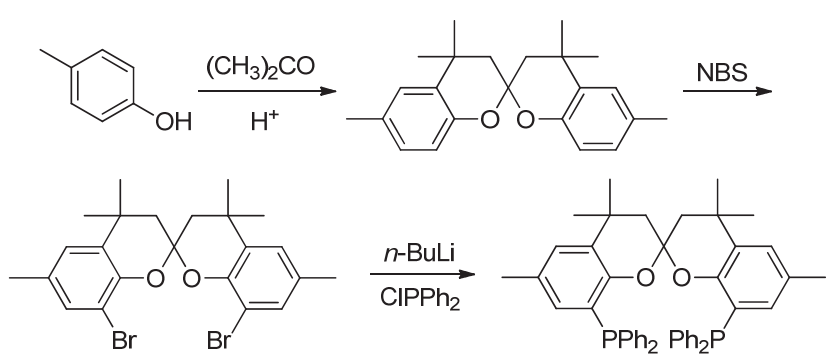

28, $80 \%$ yield

图式 9 螺二色烷骨架双膦配体 SPANphos 的合成 Scheme 9 Synthesis of bisphosphine ligand SPANphos bearing spirobichroman skeleton

对称氢化后产生的手性中心所构建的手性环境, 能够影 响螺环季碳中心的手性形成. 因此, 反式加氢的中间产 物决定着螺环中心形成时的立体选择性. 其中, Ir 的双 重催化功能是获得光学活性螺环产物的关键: $\operatorname{Ir}(\mathrm{I})$ 发挥 着碳碳双键的加氢还原的立体选择性催化作用，而 Ir(III) 是手性加氢产物中酮羰基与双酚基团螺缩酮化的 催化剂. 这种 Ir 催化的缩酮螺环的合成, 对反应物中苯 环上的取代基有着广泛的适用性，大大提高了这种螺缩 酮骨架分子结构的可修饰性，使它们具有很好的应用前 景. 基于此策略发展的手性螺环双膦配体 SKP 31, 在 $\mathrm{Pd}$ 催化的不对称烯丙位胺化反应中表现出较高的反应 活性(转化数 TON 可以达到 4750)和对映选择性, 已成 功应用于高血脂药物依折麦布的不对称合成中 ${ }^{[31]}$. 同 时, SKP 类配体 $\mathbf{L 3}$ 在钯催化的芳香胺对 Morita-BaylisHillman (MBH) 加合物 32 的不对称烯丙位胺化反应中展 
(a)<smiles>CC(C)=O</smiles><smiles>Cc1ccc(O)c(C(C)(C)O)c1</smiles><smiles>C=C(C)c1cc(C)ccc1O</smiles>

(b)<smiles>CC1=CC(=C(C)C)C(=O)C=C1</smiles>
Diels-Alder<smiles>Cc1ccc2c(c1)C(C)(C)CC(C)(c1cc(C)ccc1O)O2</smiles>
$\overrightarrow{\text { retro-Friedel-Crafts alkylation }}$<smiles>C=C1CC(C)(C)c2cc(C)ccc2O1</smiles>

(c)<smiles>C=C1CC(C)(C)c2cc(I)ccc2O/C1=C/C</smiles><smiles>Cc1ccc2c(c1)C(C)(C)CC1(CC(C)(C)c3cc(C)ccc3O1)O2</smiles>

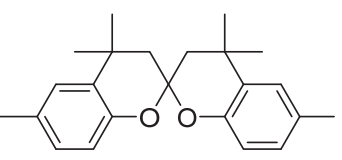

图式 10 螺二色烷骨架的构建

Scheme 10 Construction of spirobischroman skeleton

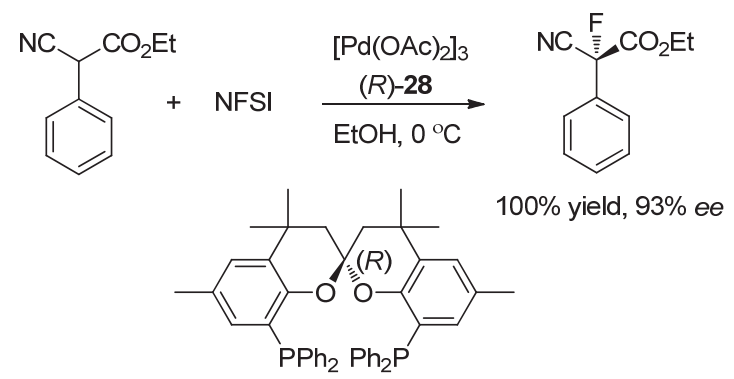

$(R)-28$<smiles>O=C1/C(=C/c2cccc(Br)c2O)CCC/C1=C\c1cccc(Br)c1O</smiles>

29
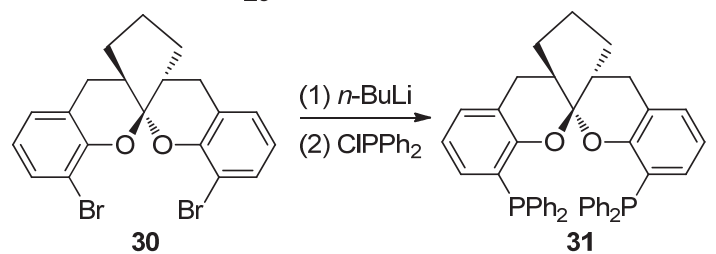

图式 11 螺二色烷骨架双膦配体 SKP 的合成

Scheme 11 Synthesis of bisphosphine ligand SKP bearing spirobichroman skeleton

现出比 SPINOL 骨架配体 SDP L4 (Eq. 4)更高的区位选 择性和对映选择性. 丁奎岭的研究发现, SKP 中磷原子 之间的距离显著大于其他配体, 这导致它在金属参与的 不对称催化反应中表现出独特的行为: 一个 $\mathrm{P}$ 原子作为 Lewis 碱与烯丙基碳原子发生作用, 而另一个 $\mathrm{P}$ 原子则 与金属结合. 重要的是, 螺缩酮结构在碱性、中性和弱 酸性条件下不仅能够保持化学稳定, 还能够能保持空间 构型的稳定. 以上利用缩酮结构构建螺环的策略为构建

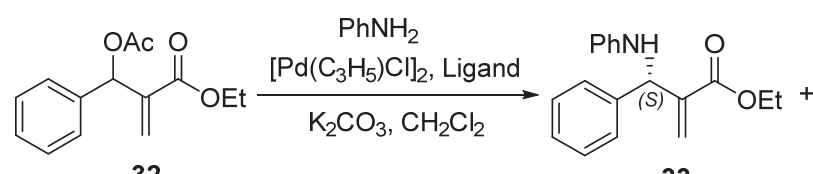

32<smiles>CCOC(=O)/C(=C/c1ccccc1)CNc1ccccc1</smiles>

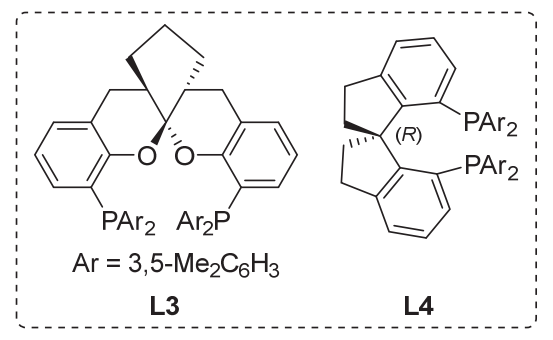

\begin{tabular}{cccc}
\hline Ligand & Yield/\% of $\mathbf{3 3}$ & ee $\%$ of $\mathbf{3 4}$ & $\mathbf{3 3 / 3 4}$ \\
\hline L3 & 89 & 96 & $92: 8$ \\
L4 & 10 & 11 & $20: 8$ \\
\hline
\end{tabular}

SPINOL 类似化合物提供了一种经济有效的合成手段， 对通过设计新型骨架来扩大螺环配体在催化不对称反 应中的适用范围具有启示作用.

2018 年, Nagorny 课题组 ${ }^{[32]}$ 利用分子内的缩酮化反 应合成了新型的具有 $C_{2}$ 对称性的螺环骨架的 SPIROL 36 (Scheme 12). 羟基保护的 3-羟基苯甲醛的高度对映 选择性烷基化被认为是手性诱导螺缩酮结构形成的关 键. 针对不同的酚差基保护基, Nagorny 课题组使用了 不同的手性氨基醇配体来完成不对称烷基化反应(Table 1). 在叔丁醇锂的作用下, 茮基醇迅速完成了与碳酸二 
乙酯的酯交换以及螺环化, 得到 $(R, S, S)$-构型的螺环产

物 35. 计算表明螺环中心手性不同的非对映异构体 $(S, S, S)-36$ 、 $(R, S, S)-36$ 的能量差异极小, 仅 $4.2 \mathrm{~kJ} / \mathrm{mol}$ (在 气相状态下预测). 因此, 在酸性条件下去除 35 中的酚 羟基保护基时, 必然会导致 36 中螺环中心的构型发生 互变. 虽然通过控制酸性可以实现某种螺环中心构型的 产物作为优势产物, 但是两种构型的螺缩酮仍然是混合 的, 并且很难分离. 在 7,7'-位酚羟基上引入三氟甲磺酰 基后, 增加了位阻, 提高了两种构型非对映异构体的转 变能垒, 通过传统色谱方法实现 $(R, S, S)-\mathbf{3 7}$ 与 $(S, S, S)-\mathbf{3 7}$ 较好的分离. Nagorny 课题组 ${ }^{[32}$ 还提出了一种适合大量 生产的拆分方法: 通过钯催化, 非对映异构体混合物 37 与二苯基氧化膦发生偶联反应, 可实现大剂量下的动力 学拆分. 动力学拆分后获得的单膦化合物 38 可以进一 步加工为双膦配体 SPIRAP $(S, S, S)-39$, 而分离得到的 $(R, S, S)-37$ 也可以转化为 $(R, S, S)-39$. 这种合成方式, 不 仅实现了大规模快速获得手性 SPIRAP, 也为类 SPINOL 骨架的构建带来了新思路. 39 已被证明是铱催化的肉桂 醇不对称甲基化等反应中的一种有效配体, 对于不对称 催化有重要价值. 之后研究发现, $(R, S, S)-39$ 与 $(S, S, S)-\mathbf{3 9}$ 在催化性能方面存在较大的差别. $(S, S, S)$-39 的催化行为 与传统螺二氢狮骨架的 $(S)-S D P$ 更相似. 晶体结构分析 发现, $(R, S, S)-39$ 分子中的乙基破坏了苯环之间的 $\pi$ 堆积, 苯环之间的二面角增大. 这种性能调节方式, 对于开发 螺环配体具有启示意义. 并且, 在催化同一反应时
$(S, S, S)$-39 与 $(R, S, S)-39$ 表现出相反的对映选择性. 这种 互补的催化效果使得 SPIRAP 39 在催化反应中具有很 大的开发潜力.

表 1 用作 SPIROL 36 原料的手性苄醇的不对称合成 Table 1 Asymmetric synthesis of starting chiral benzyl alcohols for SPIROL 36

\begin{tabular}{cccc}
\hline $\mathrm{R}^{a}$ & Ligand $^{b}$ & Yield/\% & $e e / \%$ \\
\hline $\mathrm{MOM}$ & L5 & 97 & 94 \\
$\mathrm{Bn}$ & L5 & 82 & 91 \\
$\mathrm{MOM}$ & L6 & 97 & 99 \\
$\mathrm{Bn}$ & L6 & 99 & 98 \\
$\mathrm{BOM}$ & L6 & 91 & 99 \\
\hline
\end{tabular}

${ }^{a}$ MOM: methoxymethyl, Bn: benzyl, BOM: benzyloxymethyl. ${ }^{b}$ Using the enantiomer of $\mathbf{L 5}$ or the diastereomer of $\mathbf{L 6}$, the enantiomer of chiral benzyl alcohol was obtained.

2018 年，孙兴文课题组 ${ }^{[33]}$ 也合成了含缩酮结构的 螺环骨架双膦配体(Eq. 5), 通过不对称烷基化反应和硅 胶柱色谱分离得到了配体 $(R, S, S)-\mathbf{4 0 a}$ 和 $(S, S, S)-\mathbf{4 0 b}$. 在 铑催化 $\alpha$-脱氢氨基酸酯的不对称氢化反应中, 配体 $\mathbf{4 0}$ 展现出比 SPINOL 骨架的配体 $(R)$-SDP (L4)更好的对映 选择性: $(R)-\mathrm{SDP}$ 配体催化下只能得到几乎消旋的产物 $(-10 \% e e)$, 而 $40 \mathrm{a}$ 和 $40 \mathrm{~b}$ 对映选择性分别达到了 $-65 \%$ $e e$ 和 $94.5 \% e e$. 通过设计只有一个 3-位甲基取代的骨架 (L7), 孙兴文发现甲基取代结构不仅能够帮助构建含有 两个手性中心的非对映异构体以实现便捷的拆分, 而且 对于配体的性能有着一定的影响.

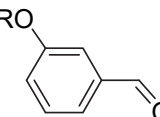<smiles>[R6]Oc1cccc([C@@H](O)CC)c1</smiles>

$\mathrm{R}=\mathrm{Bn}$

$\mathrm{R}=\mathrm{MOM}$

$\mathrm{R}=\mathrm{BOM}$
$\mathrm{OH}$<smiles>[R]Oc1cccc2c1C1(O[C@H]2C)O[C@H](CC)c2cccc(O[R])c21</smiles>

$(R, S, S)-35$

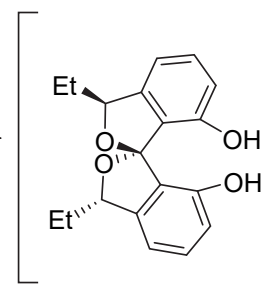

$(R, S, S)-36$

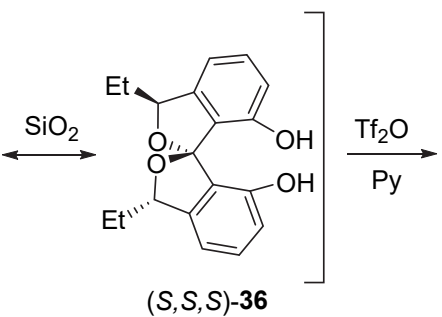

$(S, S, S)-36$

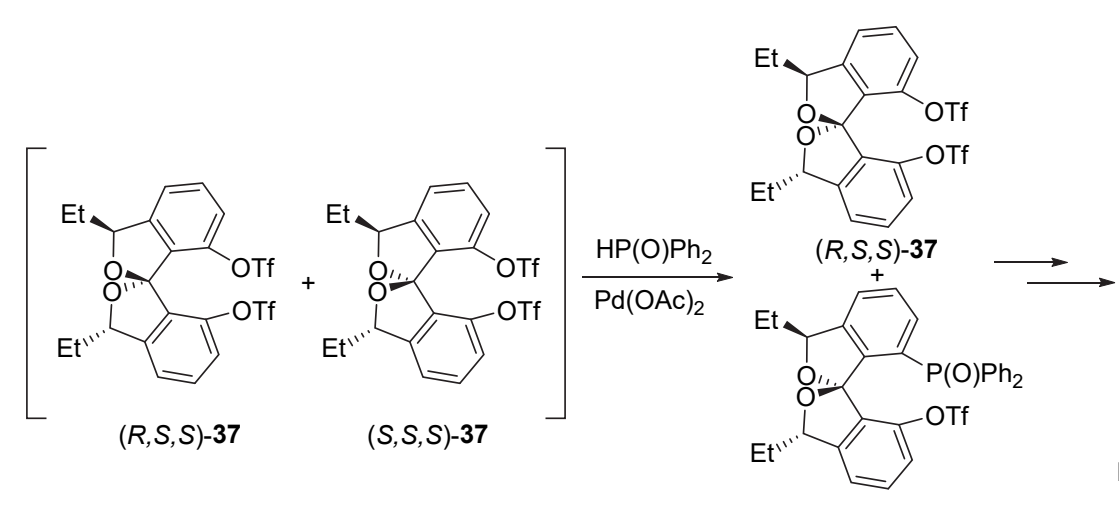

$(S, S, S)-38$

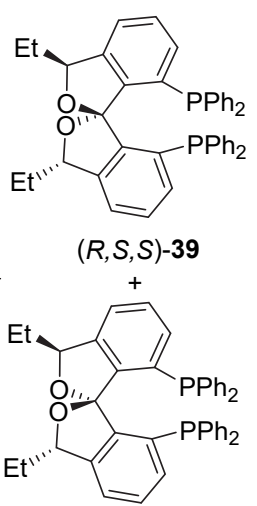

$(S, S, S)-\mathbf{3 9}$

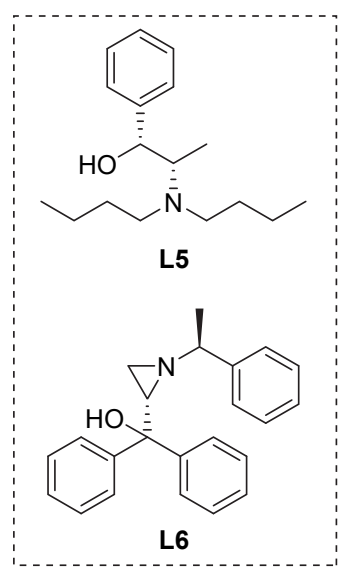

图式 12 螺缩酮骨架化合物的不对称合成

Scheme 12 Asymmetric synthesis of compounds bearing spiro ketal skeleton 


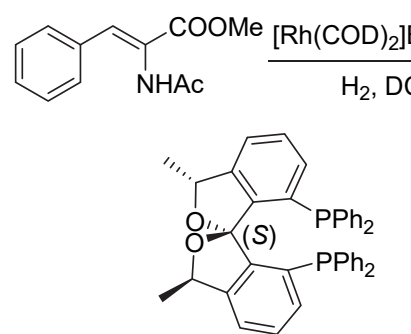

$(R, S, S)-40 a$

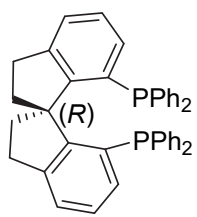

L4
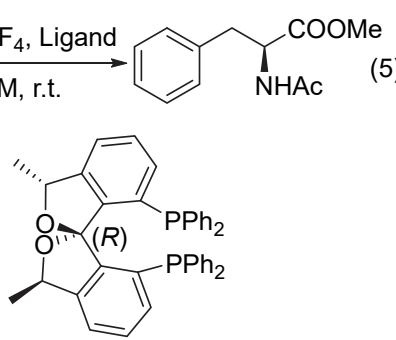

$(S, S, S)-40 b$

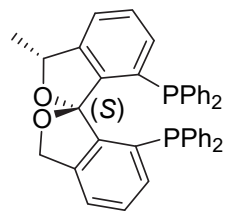

L7

\begin{tabular}{cccc}
\hline Ligand & Time/h & Yield $/ \%$ & ee/\% \\
\hline 40a & 3 & 100 & 94.5 \\
40b & 3 & 100 & -65 \\
L4 & 24 & 10 & -10 \\
L7 & 3 & 100 & 88 \\
\hline
\end{tabular}

\section{2 螺吲哚酮骨架的分子}

2012 年, 赵康课题组 ${ }^{[34]}$ 报道了有机碘试剂催化下 $\mathrm{sp}^{2}$ 与 $\mathrm{sp}^{3}$ 碳的氧化偶联反应, 由苯胺成功合成了具有 $C_{2}$ 对称结构的螺吲哚酮骨架的化合物 41 (Eq. 6). 2014 年, 龚流柱等 ${ }^{[35]}$ 在赵康的工作基础上, 利用手性高价碘试 剂实现了分子内对映选择性 $\mathrm{C}-\mathrm{H} / \mathrm{C}-\mathrm{H}$ 氧化偶联反应, 得到了一系列具有旋光活性的螺吲哚酮骨架的产物(Eq. 7). 研究表明，2-碘代间苯二酚衍生的手性催化剂分子 中脯氨酸甲酯结构在实现高对映选择性诱导时发挥了 关键作用, $N^{l}, N^{3}$-二苯基丙二酰胺反应物分子中苯环上 的取代基对产率和光学纯度有着不同程度的影响, 4-位 强给电子基团会很大程度降低螺环产物的产率和对映 选择性. 2019 年, 蔡倩课题组 ${ }^{[36]}$ 报道了铜配合物催化下 对映选择性合成螺吲哚酮的反应，扩展了芳环上取代基 的种类, 合成了多种芳环结构的螺吲哚酮类产物(Eq. 8).<smiles>CN(C(=O)CC(=O)N(C)c1ccccc1)c1ccccc1</smiles><smiles>CN1C(=O)C2(C(=O)N(C)c3ccccc32)c2ccccc21</smiles>

41

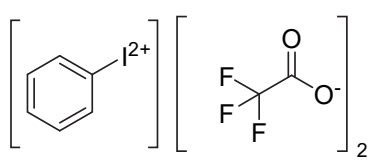

C4

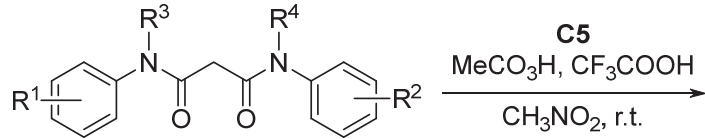<smiles>[R1]CC1(C(=O)N([R])c2ccccc2)C(=O)N([R3])c2ccccc21</smiles><smiles>[R]C(=O)C(C)Oc1cccc(O[C@H](C)C([R])=O)c1I</smiles><smiles>CN(C(=O)CC(=O)N(C)c1ccccc1I)c1ccccc1I</smiles>

(S)-41<smiles>CNC(c1ccccc1)C(c1ccccc1)N(C)C</smiles>

L
以上的研究没有将螺吲哚酮发展为手性有机配体 的骨架. 2020 年，张健存、王仲清等 ${ }^{[37]}$ 设计合成了一系 列螺吲哚酮骨架的 SPINOL 类似物 SBIDOL. 使用 $L$-氯 甲酸薄荷酯(D1)进行拆分后，经过肼解、氢解反应，分 别得到 DM-SBIDOL $(R)-42$ 和 $(S)$-42 (Scheme 13).

2020 年, 汪君课题组 ${ }^{[38]}$ 由 43 出发合成并分离了第 一例 $a z a$-SPINOL 44, 按起始反应物计, 总收率达到了 $22 \%$ (Scheme 14). $a z a-\mathrm{SPINOL}$ 分子中螺环结构合成的 关键在于对甲基苯磺酸催化的傅-克反应, 这一步达到 了 $94 \%$ 的产率. 利用氯甲酸薄荷酯(D1)拆分外消旋产物 能够达到良好的效果. 汪君课题组还发展了以此骨架为 基础的单磷配体 L8 和双膦配体 L9 (Eq. 9), 并将其应用 在双烯丙基酰胺与 NBS 的去对称化反应中，以 $55 \%$ 的 收率和 $72 \% e e$ 的对映选择性得到目标产物(Eq. 7). $a z a-$ SPINOL 骨架的配体在催化反应中的潜力值得进一步探 索.

\section{3 硅中心 SPINOL 类似物}

手性有机配体结构的变化会影响其催化活性和立 体选择性. 相比于碳原子, 硅原子具有更大的原子半径, $\mathrm{Si}-\mathrm{C}$ 键比 $\mathrm{C}-\mathrm{C}$ 键更长. 用 $\mathrm{Si}$ 代替 $\mathrm{C}$, 对空间结构的 调控可能使配体展现出与传统 SPINOL 骨架配体不同的 催化性能. 


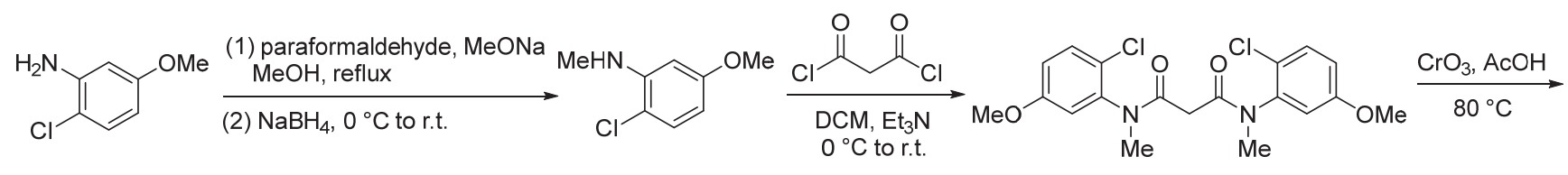<smiles>COc1ccc(Cl)c(N(C)C(=O)C(=O)N(C)c2c(Cl)ccc(OC)c2N(C)C(=O)C(=O)N(C)c2cc(OC)ccc2Cl)c1</smiles>

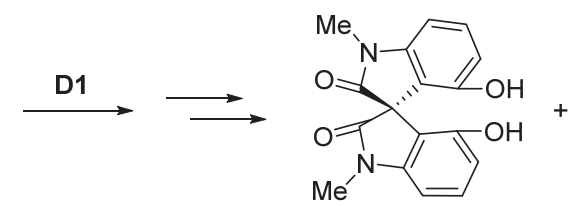

(R) -42

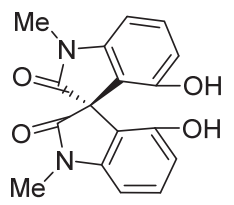

(S)-42

DM-SBIDOL

图式 13 SBIDOL 的合成与拆分

Scheme 13 Synthesis and resolution of SBIDOL

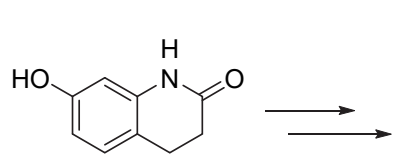

43<smiles>COc1ccc2c(c1)N(C(=O)C(=O)C(=O)N1CCCc3ccc(F)cc31)CCC2</smiles>
$\underset{\mathrm{DCE}, 140^{\circ} \mathrm{C}}{\stackrel{\mathrm{TsOH}}{(0.2 \text { equiv. })}}$<smiles>O=C1C2c3c(ccc4c3N1CCC4)C(F)(F)C(=O)N1CCCc3ccc(O)cc3C21C(=O)Oc1ccccc1</smiles><smiles>O=C1N2CCCc3ccc(O)c(c32)C12C(=O)N1CCCc3ccc(O)c2c31</smiles>

rac-44 $22 \%$ yield

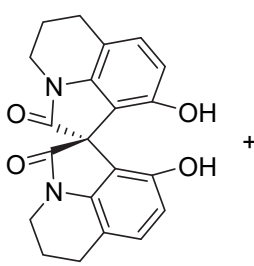

(S)-44

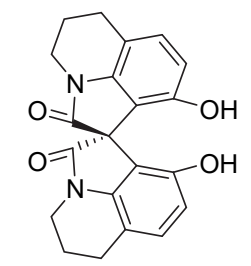

$(R)-44$

图式 14 氮杂螺二氢狮二酚类似物的合成及拆分

Scheme 14 Synthesis and resolution of $a z a$-SPINOL analogue

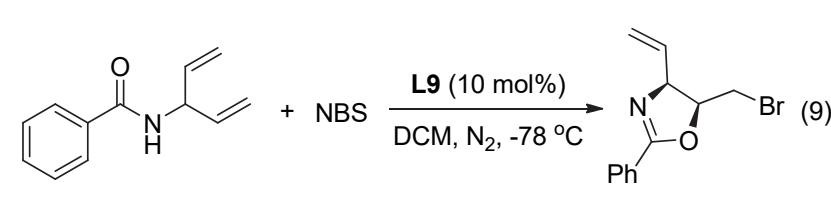
$55 \%$ yield, $72 \%$ ee

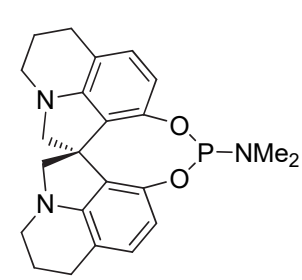

L8

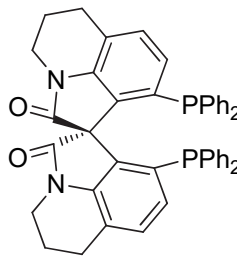

L9
2013 年, Takai 课题组 ${ }^{[39]}$ 报道了利用 $\mathrm{Rh}$ 催化 $\mathrm{C}$ - $\mathrm{Si}$ 键形成的双脱氢环化反应, 高对映选择性地合成了硅中 心的螺二芴骨架化合物 45, 发现 $[\mathrm{RhCl}(\operatorname{cod})]_{2}(\operatorname{cod}=1,5-$ 环辛二烯)和 $C_{2}$ 对称的手性双膦配体 (R)-BINAP 对构建 四芳基硅手性中心是有效的(Scheme 15a). 2020 年, 王 鹏课题组 ${ }^{[40]}$ 报道了 $\mathrm{Rh}$ 催化高效合成以硅为中心的螺环 骨架分子 SPSiOL (46, Scheme 15b)的反应, 初步展示了 SPSiOL 骨架的配体在不对称合成中的应用潜力. 王鹏 课题组利用 $(R, R)$-Et-DuPhos 或 $(R, R)$-QuinoxP 作为手性 配体，首次用铑催化的不对称双加氢反应实现了硅中心 的螺二氢狮骨架化合物的高对映选择性合成, 达到了 $98 \% e e$ 值. $\mathrm{C}=\mathrm{C}$ 与硅中心的不对称加氢偶联是高对映 选择性合成的关键, 并且不同取代基的螺环产物都具有 
良好的产率和对映选择性, 能够在大于 $10 \mathrm{~g}$ 的量级上获 得理想的反应效果. 反应底物的多样性使得硅中心螺环 骨架容易转化为多种手性配体. 王鹏课题组进一步开发 了单膦配体 SPSiPhos (L10), 发现它在不对称氢化反应 中有着较高的对映选择性(Eq. 10), 而且在分子内氢胺 化反应中比传统 SPINOL 骨架配体 L11 展现出更温和的 反应条件和更高的反应效率(Eq. 11).

(a)<smiles>COc1ccc(-c2ccccc2Sc2ccccc2-c2ccc(OC)cc2)cc1</smiles><smiles>c1ccc(-c2ccc3ccccc3c2-c2c(P(c3ccccc3)c3ccccc3)ccc3ccccc23)cc1</smiles>

$(R)$-BINAP

(b)
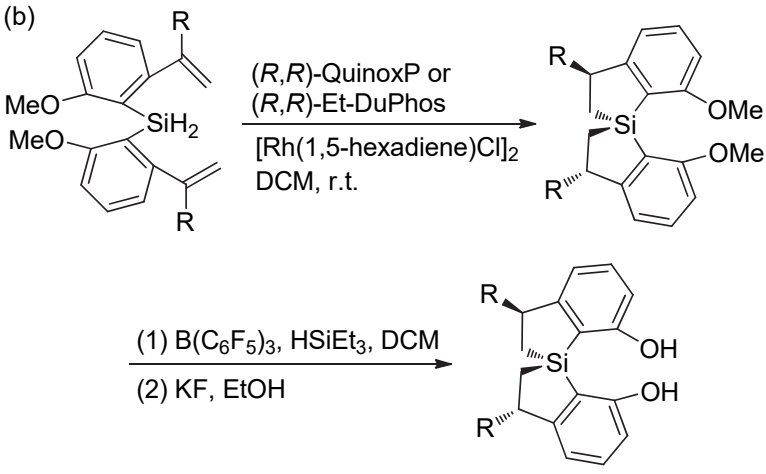

46<smiles>CCC1CCC(CC)P1c1ccccc1P1C(CC)CCC1CC</smiles>

$(R, R)$-Et-DuPhos

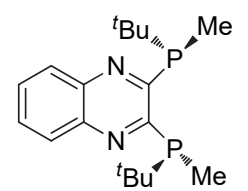

$(R, R)$-QuinoxP
图式 15 硅中心 SPINOL 类似物的不对称催化合成 Scheme 15 Catalytic asymmetric synthesis of silicon-centered SPINOL analogue

\section{3 总结与展望}

综述了自 1999 年 SPINOL 首次被合成与拆分以来, 以 SPINOL 为代表的一系列螺环骨架化合物的合成与拆 分, 着重描述了不同螺环骨架构建的设计思路、最优反 应条件、试剂或催化剂等, 略述了新颖螺环骨架配体的 应用, 并对各个合成方法的进步之处加以评述. 本文还 从螺环骨架构成的角度, 包括螺环上的取代基和含杂原 子的螺环, 对现有合成方法加以分类, 希望以此对相关

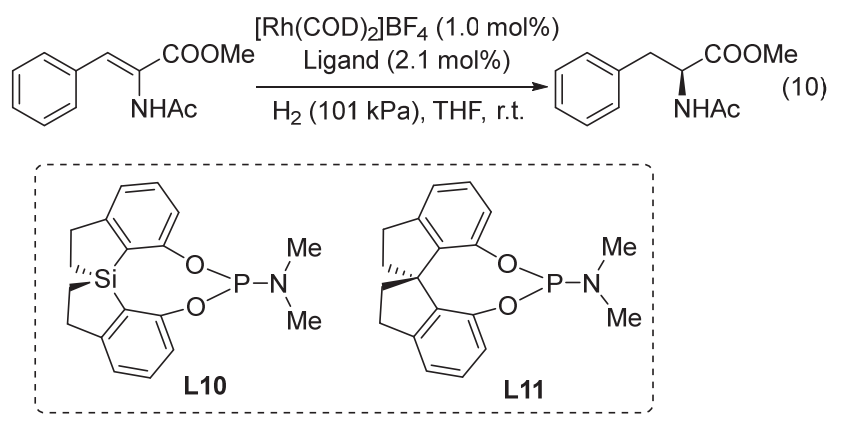

\begin{tabular}{lcc}
\hline Ligand & Yield/\% & ee/\% \\
\hline L10 & 99 & 99.8 \\
L11 & 99 & 97 \\
\hline
\end{tabular}

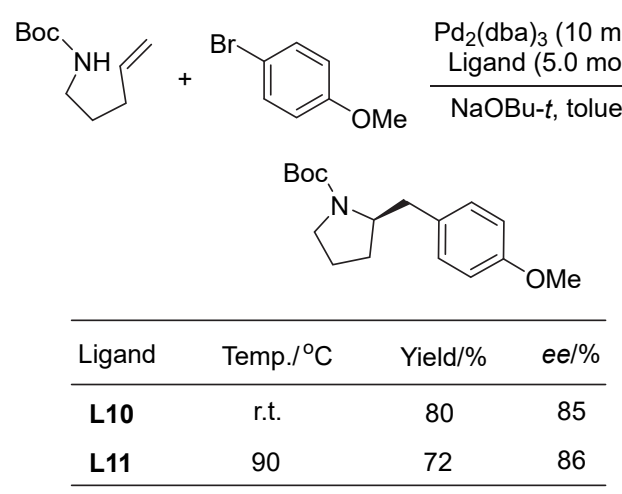

领域化学工作者在未来的研究方向有所启示. 不难看 出，以周其林、丁奎岭、林国强、张绪穆、陈新滋等为 代表的中国学者在过去 20 年里独领风骚, 彰显了中国 人在手性有机配体设计、合成及应用方面的高度智慧. 尽管 SPINOL 及螺环骨架类似物的合成与拆分方法在近 些年有了很大的进展，但更为经济、简便的合成与拆分 方式仍有待开发, 更加有效的螺环配体以及这些螺环配 体促进的新颖不对称催化反应和工业规模的应用仍然 值得期待. 我们坚信, SPINOL 衍生物及类似物作为极 具潜力的手性配体和催化剂, 有关它们的高效、精巧的 合成方法会在未来不断出现, 这会使 SPINOL 骨架成为 更具优势的手性配体结构.

致谢 本文的写作得到了南开大学化学学院谢建华教 授的指导，上海科技大学物质科学与技术学院李智教授 对 SPINOL 手性的命名规则和环己基稠合螺二氢狮二酚 化学结构式的立体示意图画法给予了十分有益的指导 和建议, 北京师范大学化学学院汪辉亮教授为我们的研 究提供了支持. 在此一并表示感谢.

\section{References}

[1] Chan, A. S. C.; Hu, W.-H.; Pai, C.-C.; Lau, C.-P.; Jiang, Y.-Z.; Mi, A.-Q.; Yan, M.; Sun, J.; Lou, R.-L.; Deng, J.-G. J. Am. Chem. Soc. 1997, 119, 9570 . 
[2] (a) Han, Z.; Wang, Z.; Zhang, X.; Ding, K. Angew. Chem., Int. Ed. 2009, 48, 5345 .

(b) Han, Z.; Wang, Z.; Zhang, X.; Ding, K. Sci. Sin.: Chem. 2010, 40, 950 (in Chinese).

(韩召斌, 王正, 张绪穆, 丁奎岭, 中国科学：化学, 2010, 40, 950.)

(c) Han, Z.; Wang, Z.; Zhang, X.; Ding, K. Chin. Sci. Bull. 2010, 55,2840 .

(d) Wang, X.; Han, Z.; Wang, Z.; Ding, K. Angew. Chem., Int. Ed. 2012, 51,936

(e) Liu, X.; Han, Z.; Wang, Z.; Ding, K. Angew. Chem., Int. Ed. 2014, 53, 1978 .

[3] (a) Zhang, B.; Hua, H.; Gao, L.; Liu, C.; Qiu, Y.; Zhou, P.; Zhou, Z.; Zhao, J.; Liang, Y. Org. Chem. Front. 2017, 4, 1376.

(b) Molteni, V.; Rhodes, D.; Rubins, K.; Hansen, M.; Bushman, F. D.; Siegel, J. S. J. Med. Chem. 2000, 43, 2031.

[4] Birman, V. B.; Rheingold, A. L.; Lam, K.-C. Tetrahedron: Asymmetry 1999, 10, 125.

[5] In their paper (Ref. [5a]) in 1956, Cahn, Ingold and Prelog considered the chirality similar to SPINOL as axial type, and defined the absolute configuration according to the rules for BINOL. In their paper (Ref. [5b]) in 1966, they definitely considered the same chirality as central type, and gave new rules to define the chirality. Since the original report on SPINOL by Birman, scholars around the world followed the new rules by Cahn, Ingold and Prelog to define the absolute configurations of spiro centers in SIPNOL and analogues, in which an axis with restricted rotation is absent. For this reason, the chiral senses of $(S)$-SPINOL and $(R)$-BINOL are the same. We think it is more reasonable to consider the chirality in SPINOL as central instead of axial type.

(a) Cahn, R. S.; Ingold, C. K.; Prelog, V. Experientia 1956, 12, 81.

(b) Cahn, R. S.; Ingold, C. K.; Prelog, V. Angew. Chem., Int. Ed. 1966, 5, 385.

(c) Favre, H. A.; Powell, W. H. Nomenclature of Organic Chemistry: IUPAC Recommendations and Preferred Names, 2013 Royal Society of Chemistry, Cambridge, 2014, p. 1261.

(d) Lin, G.-Q.; Sun, X.-W.; Chen, Y.-Q.; Li, Y.-M.; Chan, A. S. C. Chiral Synthesis-Asymmetric Reactions and Their Applications, 5th ed., Science Press, Beijing, 2013, p. 9 (in Chinese).

(林国强, 孙兴文, 陈耀全, 李月明, 陈新滋, 手性合成一一不对 称反应及其应用, 第五版, 科学出版社, 北京, 2013, p. 9.)

[6] (a) Hu, A.-G.; Fu, Y.; Xie, J.-H.; Zhou, H.; Wang, L.-X.; Zhou, Q.-L. Angew. Chem., Int. Ed. 2002, 41, 2348.

(b) Fu, Y.; Xie, J.-H.; Hu, A.-G.; Zhou, H.; Wang, L.-X.; Zhou, Q.-L. Chem. Commun. 2002, 480.

[7] (a) Xie, J.-H.; Zhou, Q.-L. Acc. Chem. Res. 2008, 41, 581.

(b) Ding, K.; Han, Z.; Wang, Z. Chem. Asian. J. 2009, 4, 32.

(c) Xie, J.-H.; Zhou, Q.-L. Acta Chim. Sinica 2014, 72, 778 (in Chinese).

(谢建华, 周其林, 化学学报, 2014, 72, 778.)

[8] (a) Xu, F.; Huang, D.; Han, C.; Shen, W.; Lin, X.; Wang, Y. J. Org. Chem. 2010, 75, 8677 .

(b) Rahman, A.; Lin, X. Org. Biomol. Chem. 2018, 16, 4753.

[9] Li, Z.; Liang, X.-M.; Wu, F.; Wan, B.-S. Tetrahedron: Asymmetry 2004, 15, 665

[10] (a) Chen, Y.; Yekta, S.; Yudin, A. K. Chem. Rev. 2003, 103, 3155.

(b) Brunel, J. M. Chem. Rev. 2005, 105, 857.

(c) Bringmann, G.; Gulder, T.; Gulder, T. A. M.; Breuning, M. Chem. Rev. 2011, 111, 563.

[11] Zhang, J.-H.; Liao, J.; Cui, X.; Yu, K.-B.; Zhu, J.; Deng, J.-G.; Zhu, S.-F.; Wang, L.-X.; Zhou, Q.-L.; Chung, L.-W.; Ye, T. Tetrahedron: Asymmetry 2002, 13, 1363.

[12] Lu, S.; Poh, S. B.; Zhao, Y. Angew. Chem., Int. Ed. 2014, 53, 11041.
[13] Huang, D. Ph.D. Dissertation, Zhejiang University, Zhejiang, 2013 (in Chinese). (黄丹, 博士论文, 浙江大学, 浙江, 2013.)

[14] Li, S.; Zhang, J.-W.; Li, X.-L.; Cheng, D.-J.; Tan, B. J. Am. Chem. Soc. 2016, 138, 16561

[15] (a) Huang, D.; Li, X.; Xu, F.; Li, L.; Lin, X. ACS Catal. 2013, 3, 2244.

(b) Li, X.; Chen, D.; Gu, H.; Lin, X. Chem. Commun. 2014, 50, 7538.

[16] Venugopal, M.; Elango, S.; Parthiban, A.; Eni. Tetrahedron: Asymmetry 2004, 15, 3427.

[17] Zheng, Z.; Cao, Y.; Zhu, D.; Wang, Z.; Ding, K. Chem.-Eur. J. 2019, 25, 9491.

[18] Zheng, Z.; Cao, Y.; Chong, Q.; Han, Z.; Ding, J.; Luo, C.; Wang, Z.; Zhu, D.; Zhou, Q.-L.; Ding, K. J. Am. Chem. Soc. 2018, 140, 10374.

[19] Yin, L.; Xing, J.; Wang, Y.; Shen, Y.; Lu, T.; Hayashi, T.; Dou, X. Angew. Chem., Int. Ed. 2019, 58, 2474.

[20] Chang, S.; Wang, L.; Lin, X. Org. Biomol. Chem. 2018, 16, 2239.

[21] (a) Gu, H.; Han, Z.; Xie, H.; Lin, X. Org. Lett. 2018, 20, 6544. (b) Lin, X.; Han, Z. CN 109020788, 2018 (in Chinese).

[22] Zhou, Q.; Pan, R.; Shan, H.; Lin, X. Synthesis 2019, 51, 557.

[23] Wang, L.; Zhong, J.; Lin, X. Angew. Chem., Int. Ed. 2019, 58, 15824

[24] Smith, L. K.; Baxendale, I. R. Org. Biomol. Chem. 2015, 13, 9907.

[25] (a) Ding, A.; Meazza, M.; Guo, H.; Yang, J.-W.; Rios, R. Chem. Soc. Rev. 2018, 47, 5946.

(b) Xu, P.; Yu, J.; Chen, C.; Cao, Z.; Zhou, F.; Zhou, J. ACS Catal. 2019, 9, 1820.

[26] Chen, G.; Lin, B.; Huang, J.; Zhao, L.; Chen, Q.; Jia, S.; Yin, Q.; Zhang, X. J. Am. Chem. Soc. 2018, 140, 8064.

[27] Yang, W.; Wang, X.; Jin, X.; Sun, H.; Guo, R.; Xu, W.; Cai, Q. Adv. Synth. Catal. 2019, 361, 562.

[28] Freixa, Z.; Beentjes, M. S.; Batema, G. D.; Dieleman, C. B.; van Strijdonck, G. P. F.; Reek, J. N. H.; Kamer, P. C. J.; Fraanje, J.; Goubitz, K.; van Leeuwen, P. W. N. M. Angew. Chem., Int. Ed. 2003, 42, 1284.

[29] Freixa, Z.; Kamer, P. C. J.; Lutz, M.; Spek, A. L.; van Leeuwen, P. W. N. M. Angew. Chem., Int. Ed. 2005, 44, 4385.

[30] Jacquet, O.; Clément, N. D.; Blanco, C.; Belmonte, M. M.; BenetBuchholz, J.; van Leeuwen, P. W. M. N. Eur. J. Org. Chem. 2012 , 4844.

[31] (a) Wang, X.; Meng, F.; Wang, Y.; Han, Z.; Chen, Y.; Liu, L.; Wang, Z.; Ding, K. Angew. Chem., Int. Ed. 2012, 51, 9276.

(b) Wang, X.; Guo, P.; Han, Z.; Wang, X.; Wang, Z.; Ding, K. J. Am. Chem. Soc. 2014, 136, 405.

[32] Argüelles, A. J.; Sun, S.; Budaitis, B. G.; Nagorny, P. Angew. Chem., Int. Ed. 2018, 57, 5325.

[33] Huang, J.; Hong, M.; Wang, C.; Kramer, S.; Lin, G.; Sun, X. J. Org. Chem. 2018, 83, 12838.

[34] Wang, J.; Yuan, Y.; Xiong, R.; Zhang-Negrerie, D.; Du, Y.; Zhao, K. Org. Lett. 2012, 14, 2210.

[35] Wu, H.; He, Y.-P.; Xu, L.; Zhang, D.-Y.; Gong, L.-Z. Angew. Chem., Int. Ed. 2014, 53, 3466.

[36] Liu, T.; Feng, J.; Chen, C.; Deng, Z.; Kotagiri, R.; Zhou, G.; Zhang, X.; Cai, Q. Org. Lett. 2019, 21, 4505.

[37] Sun, G.; Wang, Z.; Luo, Z.; Lin, Y.; Deng, Z.; Li, R.; Zhang, J. J. Org. Chem. 2020, 85, 10584.

[38] Guo, W.; Liu, Q.; Jiang, J.; Wang, J. Org. Lett. 2020, 22, 3110.

[39] Kuninobu, Y.; Yamauchi, K.; Tamura, N.; Seiki, T.; Takai, K. Angew. Chem., Int. Ed. 2013, 52, 1520.

[40] Chang, X.; Ma, P.; Chen, H.; Li, C.; Wang, P. Angew. Chem., Int. Ed. 2020, 59, 8937. 